\title{
ON LIMIT PROBLEMS ASSOCIATED WITH SOME INEQUALITIES
}

\author{
by P. H. DIANANDA
}

(Received 15 December, 1971)

1. Let $\left\{a_{n}\right\}$ be a sequence of non-negative real numbers. Suppose that

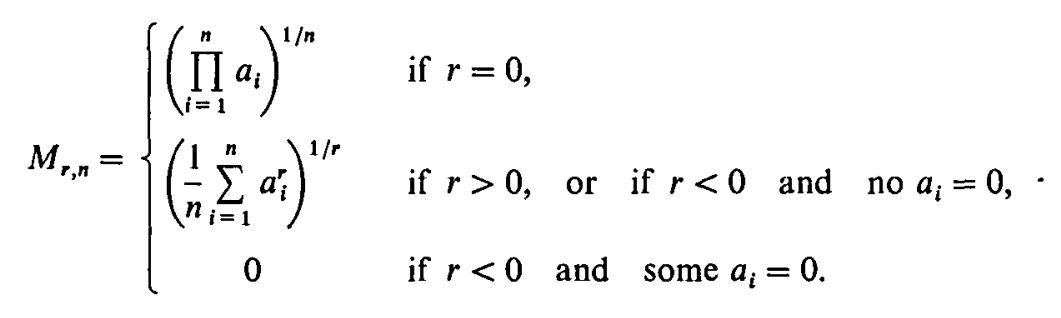

Then $M_{1, n}$ is the arithmetic mean, $M_{0, n}$ the geometric mean, and $M_{r, n}$ the generalized mean of order $r$, of $a_{1}, a_{2}, \ldots, a_{n}$. By a result of Everitt [1] and McLaughlin and Metcalf [5], $\left\{n\left(M_{r, n}-M_{s, n}\right)\right\}$, where $r \geqq 1 \geqq s$, is a monotonic increasing sequence. It follows that this sequence tends to a finite or an infinite limit as $n \rightarrow \infty$. Everitt $[2,3]$ found a necessary and sufficient condition for the finiteness of this limit in the cases $r, s=1,0$ and $r \geqq 1>s>0$. His results are included in the following theorem.

THEOREM 1. Let $\left\{a_{n}\right\}$ be a sequence of non-negative real numbers, and either $r>1 \geqq s$ or $r \geqq 1>s$. Then $n\left(M_{r, n}-M_{s, n}\right)$ tends to a finite or an infinite limit as $n \rightarrow \infty$. This limit is finite if and only if (i) every $a_{n}=0$, or (ii) $r=1$ and $a_{1}+a_{2}+\ldots$ is finite, or (iii) $\sum_{n=1}^{\infty}\left(a_{n}-\alpha\right)^{2}$ is finite for some finite positive $\alpha$, and either $s \leqq 0$ and every $a_{n}>0$, or $s>0$.

The proof, which we omit, is a straightforward modification of the proofs of the cases $r, s=1,0$ and $r \geqq 1>s>0$ (see [2,3]).

Let now the $a_{n}$ be all positive. Then, by a result of McLaughlin and Metcalf [5], the sequence $\left\{\left(M_{r, n} / M_{s, n}\right)^{n}\right\}$, where $r \geqq 0 \geqq s$, is monotonic increasing. It follows that this sequence tends to a finite or an infinite limit as $n \rightarrow \infty$. The question naturally arises as to what are the conditions under which this limit is finite. It is our main purpose in this paper to answer this question. Our answer is contained in

THEOREM 2. Let $\left\{a_{n}\right\}$ be a sequence of positive numbers, and either $r>0 \geqq s$ or $r \geqq 0>s$. Then $\left(M_{r, n} / M_{s, n}\right)^{n}$ tends to a finite or an infinite limit as $n \rightarrow \infty$. This limit is finite if and only if $\sum_{n=1}^{\infty}\left(a_{n}-\alpha\right)^{2}$ is finite for some finite positive $\alpha$.

We prove this result in $\S 4$. We discuss some results allied to those stated above in $\S \S 2$ and 3 . These allied results are used in our proof in $\S 4$. We state another result in $\S 5$. 
2. Let the $a_{n}$ be non-negative and suppose that $r>s>1$ or $1>r>s$. Then the sequence $\left\{n\left(M_{r, n}-M_{s, n}\right)\right\}$ need not be monotonic increasing. This can be seen as follows.

First, let $r>s>1,0<\delta<1, a_{1}=a_{2}=\ldots=a_{n-2}=1, a_{n-1}=(1+\delta)^{1 / r}, a_{n}=(1-\delta)^{1 / r}$ and $a_{n+1}=1$. Then

$$
(n+1)\left(M_{r, n+1}-M_{s, n+1}\right) \geqq n\left(M_{r, n}-M_{s, n}\right)
$$

is equivalent to $n M_{s, n}+1 \geqq(n+1) M_{s, n+1}$. This is equivalent to $M_{1, n+1} \geqq M_{s, n+1}$, where now $a_{1}=a_{2}=\ldots=a_{n}=M$ and $a_{n+1}=1$, with $M=n^{-1 / s}\left\{n-2+(1+\delta)^{s / r}+(1-\delta)^{s / r}\right\}^{1 / s}$. The last inequality is false since $M<1$ and $s>1$ [4, p. 26]. Thus (1) is false.

Next, let $1>r>s, 0<\delta<1, a_{1}=a_{2}=\ldots=a_{n-2}=1, a_{n-1}=(1+\delta)^{1 / s}, a_{n}=(1-\delta)^{1 / s}$ and $a_{n+1}=1$. Then we can see, as in the last paragraph, that (1) is false.

Thus the sequence $\left\{n\left(M_{r, n}-M_{s, n}\right)\right\}$ is not necessarily monotonic increasing if $r>s>1$ or $1>r>s$. The question still arises as to whether Theorem 1 can be proved with " $r>s$ ", in place of "either $r>1 \geqq s$ or $r \geqq 1>s$ ". I have not been able to settle this question. However, we can prove

THEOREM 3. Let $\left\{a_{n}\right\}$ be a sequence of non-negative real numbers, $a_{n} \rightarrow \alpha$ as $n \rightarrow \infty, \alpha$ be finite and positive, and $r>s$. Then $n\left(M_{r, n}-M_{s, n}\right)$ tends to a finite or an infinite limit as $n \rightarrow \infty$. This limit is finite if and only if (i) $r \leqq 0$ and some $a_{n}=0$, or (ii) $\sum_{n=1}^{\infty}\left(a_{n}-\alpha\right)^{2}$ is finite, and either $s \leqq 0$ and every $a_{n}>0$, or $s>0$.

The proof, which we omit, is a straightforward modification of the proof of the corresponding part of Theorem 1 .

3. Let the $a_{n}$ be positive and suppose that $r>s>0$ or $0>r>s$. Then the sequence $\left\{\left(M_{r, n} \mid M_{s, n}\right)^{n}\right\}$ need not be monotonic increasing. This can be seen as follows.

First, let $r>s>0,0<\delta<1, a_{1}=a_{2}=\ldots=a_{n-2}=1, a_{n-1}=(1+\delta)^{1 / r}, a_{n}=(1-\delta)^{1 / r}$ and $a_{n+1}=1$. Then

$$
\left(M_{r, n+1} / M_{s, n+1}\right)^{n+1} \geqq\left(M_{r, n} / M_{s, n}\right)^{n}
$$

is equivalent to $M_{s, n}^{n s} \geqq M_{s, n+1}^{(n+1) s}$, or to $(1-\varepsilon / n)^{n} \geqq(1-\varepsilon /(n+1))^{n+1}$, where

$$
\varepsilon=2-(1+\delta)^{s / r}-(1-\delta)^{s / r} \text {. }
$$

The last inequality is false [4, p. 37] since $0<\varepsilon<1$. Thus (2) is false.

Next, let $0>r>s, 0<\delta<1, a_{1}=a_{2}=\ldots=a_{n-2}=1, a_{n-1}=(1+\delta)^{1 / s}, a_{n}=(1-\delta)^{1 / s}$ and $a_{n+1}=1$. Then we can see, as in the last paragraph, that (2) is false.

Thus the sequence $\left\{\left(M_{r, n} / M_{s, n}\right)^{n}\right\}$ is not necessarily monotonic increasing if $r>s>0$ or $0>r>s$. Despite this we can prove the following analogue of Theorem 3.

THEOREM 4. Let $\left\{a_{n}\right\}$ be a sequence of positive numbers, $a_{n} \rightarrow \alpha$ as $n \rightarrow \infty, \alpha$ be finite and positive, and $r>s$. Then $\left(M_{r, n} / M_{s, n}\right)^{n}$ tends to a finite or an infinite limit as $n \rightarrow \infty$. This limit is finite if and only if $\sum_{n=1}^{\infty}\left(a_{n}-\alpha\right)^{2}$ is finite. 
Proof. We note that, since $r>s, M_{r, n}-M_{s, n} \geqq 0$. Since the $a_{n}>0$ and $a_{n} \rightarrow \alpha>0$ as $n \rightarrow \infty$, it follows that $M_{s, n} \rightarrow \alpha>0$ as $n \rightarrow \infty$.

Suppose that $\sum_{n=1}^{\infty}\left(a_{n}-\alpha\right)^{2}$ is finite. Then, by Theorem $3, n\left(M_{r, n}-M_{s, n}\right)$ tends to a finite limit $L$, say, as $n \rightarrow \infty$. Thus

$$
\left(\frac{M_{r, n}}{M_{s, n}}\right)^{n}=\left(1+\frac{M_{r, n}-M_{s, n}}{M_{s, n}}\right)^{n}=\left(1+\frac{L}{\alpha n}(1+o(1))\right)^{n} \rightarrow e^{L / \alpha}<\infty \quad \text { as } n \rightarrow \infty .
$$

Suppose that $\sum_{n=1}^{\infty}\left(a_{n}-\alpha\right)^{2}$ is infinite. Then, since no $a_{n}=0$, by Theorem $3, n\left(M_{r, n}-M_{s, n}\right)$ $\rightarrow \infty$ as $n \rightarrow \infty$. From this and (3),

$$
\left(\frac{M_{r, n}}{M_{s, n}}\right)^{n}>n\left(\frac{M_{r, n}-M_{s, n}}{M_{s, n}}\right) \rightarrow \infty \quad \text { as } n \rightarrow \infty .
$$

This completes the proof of Theorem 4.

4. In the proof of Theorem 2 we consider the following four cases, which exhaust all possibilities:

Case 1. $\liminf _{n \rightarrow \infty} a_{n}=0$.

Case 2. $\limsup _{n \rightarrow \infty} a_{n}=\infty$.

Case 3. $0<\lambda \equiv \liminf _{n \rightarrow \infty} a_{n}<\Lambda \equiv \limsup _{n \rightarrow \infty} a_{n}<\infty$.

Case 4. $0<\alpha \equiv \lim _{n \rightarrow \infty} a_{n}<\infty$.

Let $r>0 \geqq s$ or $r \geqq 0>s$, and

$$
\tau\left(a_{1}, a_{2}, \ldots, a_{n}\right)=\left(M_{r, n} / M_{s, n}\right)^{n} .
$$

Then, by a result of McLaughlin and Metcalf [5],

$$
\begin{aligned}
\tau\left(a_{1}, a_{2}, \ldots, a_{n}\right) & \geqq \tau\left(a_{1}, a_{2}, \ldots, a_{m}\right) \tau\left(a_{m+1}, a_{m+2}, \ldots, a_{n}\right) \\
& \geqq \tau\left(a_{1}, a_{2}, \ldots, a_{m}\right) \geqq 1,
\end{aligned}
$$

if $1 \leqq m<n$. Also, $\tau\left(a_{1}, a_{2}, \ldots, a_{n}\right)$ necessarily tends to a finite or an infinite limit as $n \rightarrow \infty$.

Case 1. In this case, there is an increasing sequence of integers $\{i(n)\}$ such that $a_{i(n)} \rightarrow 0$ as $n \rightarrow \infty$. Consequently, from (5),

$$
\tau\left(a_{1}, a_{2}, \ldots, a_{i(n)}\right) \geqq \tau\left(a_{1}, a_{i(n)}\right) .
$$


Now $M_{r, n}$ is an increasing function of $r$ for fixed $a_{1}, a_{2}, \ldots, a_{n}$. Hence, by (4) and (6), we have (i) if $r>0 \geqq s$, then

$$
\begin{aligned}
\tau\left(a_{1}, a_{2}, \ldots, a_{i(n)}\right) & \geqq\left(\frac{a_{1}^{r}+a_{i(n)}^{r}}{2}\right)^{1 / r} /\left(a_{1} a_{i(n)}\right)^{1 / 2} \\
& >2^{-1 / r}\left(a_{1} / a_{i(n)}\right)^{1 / 2} \rightarrow \infty \quad \text { as } n \rightarrow \infty
\end{aligned}
$$

and (ii) if $r \geqq 0>s$, then, in a similar way,

$$
\tau\left(a_{1}, a_{2}, \ldots, a_{i(n)}\right)>2^{1 / s}\left(a_{1} / a_{i(n)}\right)^{1 / 2} \rightarrow \infty \quad \text { as } n \rightarrow \infty .
$$

Hence, by (4) and (5), it follows that

$$
\left(M_{r, n} / M_{s, n}\right)^{n} \rightarrow \infty \quad \text { as } n \rightarrow \infty
$$

Case 2. In this case, there is an increasing sequence of integers $\{i(n)\}$ such that $a_{i(n)} \rightarrow \infty$ as $n \rightarrow \infty$. Consequently, by a modification of the argument used in Case 1, we have (7).

Case 3. In this case, there are increasing sequences of integers $\{i(n)\}$ and $\{j(n)\}$, with $i(n)<j(n)<i(n+1)$ for each $n$, such that $a_{i(n)} \rightarrow \lambda$ and $a_{j(n)} \rightarrow \Lambda$ as $n \rightarrow \infty$, where $0<\lambda<\Lambda<\infty$. Consequently, by (5),

$$
\tau\left(a_{1}, a_{2}, \ldots, a_{j(n)}\right) \geqq \prod_{t=1}^{n} \tau\left(a_{i(t)}, a_{j(t)}\right) \rightarrow \infty \quad \text { as } n \rightarrow \infty,
$$

since $\tau\left(a_{i(n)}, a_{j(n)}\right) \rightarrow \tau(\lambda, \Lambda)>1$ as $n \rightarrow \infty$. Thus we have (7).

Case 4. In this case, by Theorem 4, we have that (i) (7) holds if $\sum_{n=1}^{\infty}\left(a_{n}-\alpha\right)^{2}$ is infinite, and (ii) $\left(M_{r, n} / M_{s, n}\right)^{n}$ tends to a finite limit as $n \rightarrow \infty$ if $\sum_{n=1}^{\infty}\left(a_{n}-\alpha\right)^{2}$ is finite.

It can now be seen that Theorem 2 follows from Cases 1 to 4 .

5. We omit the proof of the following result, which we can deduce directly from Theorem 1 , by modifying the arguments used in the proof of Theorem 4 (see $\S 3$ ).

THEOREM 5. Let $\left\{a_{n}\right\}$ be a bounded sequence of non-negative real numbers, not all zero, and either $r>1 \geqq s>0$ or $r \geqq 1>s>0$. Then the conclusions of Theorem 2 are valid.

I am grateful to the referee for his helpful comments.

\section{REFERENCES}

1. W. N. Everitt, On an inequality for the generalized arithmetic and geometric means, Amer. Math. Monthly 70 (1963), 251-255.

2. W. N. Everitt, On a limit problem associated with the arithmetic-geometric mean inequality, J. London Math. Soc. 42 (1967), 712-718. 
3. W. N. Everitt, Corrigendum to [2], J. London Math. Soc. (2) 1 (1969), 428-430.

4. G. H. Hardy, J. E. Littlewood and G. Pólya, Inequalities (Cambridge, 1934).

5. H. W. McLaughlin and F. T. Metcalf, An inequality for generalized means, Pacific J. Math. 22 (1967), 303-311.

UNIVERSITY OF SINGAPORE 